\title{
Características clínicas de 47 casos de hiperparatiroidismo primario intervenidos quirúrgicamente
}

\author{
J. D. MEDIAVILLA GARCÍA, M. LÓPEZ DE LA TORRE CASARES*, \\ C. HIDALGO TENORIO, F. PERÁN MESA**, L. LEÓN RUIZ, \\ L. ALIAGA MARTÍNEZ, Ma . P. PINEL JULIÁN*** \\ Servicio de Medicina Interna. *Endocrinología. **Análisis Clínicos. Hospital Virgen de \\ las Nieves. Granada
}

\begin{abstract}
THE CLINICAL MANIFESTATIONS OF 47 PATIENTS WITH PRI MARY HYPERPARATHYROIDISM INTERVENED
\end{abstract}

\begin{abstract}
RESUMEN
Fundamento y objetivo: El hiperparatiroidismo primario (HPTP) en la mayoría de los casos es asintomático. Hoy día, se discute la necesidad de someter a estos enfermos a paratiroidectomía. En el presente estudio, pretendemos describir las características clínicas y bioquímicas de nuestra serie de HPTP, además, de describir las técnicas utilizadas en el diagnóstico y los resultados obtenidos tras la intervención quirúrgica.

Material y métodos: Hemos revisado las historias clínicas de 47 pacientes diagnosticados y operados de HPTP en nuestro centro en los últimos cinco años. Primero, recogimos las manifestaciones clínicas; segundo, los resultados bioquímicos anteriores y posteriores a la intervención quirúrgica; tercero, los datos de la ecografía paratiroidea, tomografía computerizada (TC) cervico-torácica, gammagrafía paratiroidea con 99Tc-Sestamibi y densitometría dual de rayos X ( DEXA); y por último, los resultados derivados de la intervención quirúrgica.

Resultados: Fueron intervenidos 47 pacientes de HPTP, 89,4\% mujeres, con edad media $61,4 \pm 12,8$ años. La gran mayoría se encontraban asintomáticos $(45,7 \%)$. En cuanto a las técnicas de localización, la gammagrafía con $99 \mathrm{mTc}$-sestamibi fue la que tuvo mayor sensibilidad y especificidad 75 y $100 \%$, respectivamente. Ninguno de los pacientes presentó osteoporosis mediante densitometría ósea. El principal diagnóstico fue el de adenoma $85,1 \%$. Todos los pacientes curaron tras la intervención, con la excepción de un solo caso.

Conclusión: El HPTP presenta escasos síntomas. Principalmente se trata de adenomas. La técnica de localización preoperatoria más útil es la gammagrafía. El tratamiento definitivo es la extirpación.
\end{abstract}

PALABRAS CLAVE: Hiperparatiroidismo primario. Diagnóstico del HPTP. Tratamiento del HPTP.

\begin{abstract}
Background: The primary hyperparathyroidism is usually an asymp tomatic disease. Actually, specialists are attempting to consider to what extent it can be necessary to undergo surgery in this disease. In this arti cle, we are going to describe the clinical and biochemical characteristics of our patients with primary hyperparathyroidism as well as the diagnos tic procedures and the results after operation.

Methods: Firstly, we have analysed 47 records of patients with pri mary hyperparathyroidism and intervened in our hospital during the last 5 years. Secondly, we assessed the biochemical results before and after the surgical intervention, the outcome of parathyroid echography, thora cico-cervical tomography, technietium-99-mm-sestamibi gammagraphy and bone densitometry. Finally, we followed up the assessment of the operation of the patients with hyperparathyroidism.

Results: 47 patients were intervened of hyperparathyroidism. $89.4 \%$ were women and average the age was $61.4 \pm 12.8$ years. Most of them were asymptomatic (45.7\%). The gammagraphy was the procedure with best sensibility and specificity, 75 and 100\%. Bone densitometry showed that there were no patients with diminished calcification of the bones. The principal diagnostic of pathologic anatomy was adenoma (85.1\%). Excluding one single case, the rest of patients healed of their ilness after intervention.

Conclusions: Primary hyperparathyroidism is asymptomatic in most of the cases. It is mainly an adenoma process. The best diagnostic proce dure is technietium-99-mm-sestamibi gammagraphy. The most appro priate and successful treatment is surgery.
\end{abstract}

KEY WORDS: Hyperparathyroidism treatment. Diagnostic and treat ment.

Mediavilla García JD, López de la Torre Casares M, Hidalgo Tenorio C, Perán Mesa F, León Ruiz, L, Aliaga Martínez, L, Pinel Julián MaP. Características clínicas de 47 casos de hiperparatiroidismo primario intervenidos quirúrgicamente. An Med Interna (Madrid) 2001; 18: 468-472.

\section{INTRODUCCIÓN}

El hiperparatiroidismo primario (HPTP) es un trastorno hipercalcémico que resulta de la secreción inadecuada de PTH. La mayoría de los pacientes están asintomáticos, y se diagnostican casualmente en análisis de rutina. El tratamiento definiti- vo del HPTP es la paratiroidectomía quirúrgica, sin embargo, en la actualidad, se considera un tema en debate. En el presente estudio describimos las características clínicas y bioquímicas de los pacientes intervenidos de HPTP de nuestra serie; recogemos, también, los métodos diagnósticos utilizados y los resultados obtenidos tras la intervención quirúrgica.

Trabajo aceptado: 7 de marzo de 2001

Correspondencia: C. Hidalgo Tenorio. C/. Víctor Hugo, 3. Osuna 1-2º B. 18011 Granada. 


\section{MATERIAL Y MÉTODOS}

Hemos revisado las historias clínicas de 47 pacientes diagnosticados y operados de HPTP en nuestro centro en los últimos cinco años. Se consideró que el paciente presentaba clínica cuando tenía algún síntoma relacionado con el HPTP.

Los datos bioquímicos se recogieron antes y después de la intervención. La determinación de PTH intacta plasmática se realizó mediante un método inmunoradiométrico (IRMA) (Nichols Inst. Diag. San Juan Capistrano, CA-92675) que usa 2 anticuerpos dirigidos frente a las regiones 39-84 y 1-34 respectivamente de la molécula de PTH, y 1-84 intacta (humana) como estándar. Los coeficientes de variación Inter.-intraensayo respectivos fueron del 6,1 y $5,6 \%$; la sensibilidad era de $1 \mathrm{pg} / \mathrm{mL}$ y los valores de referencia $20-70 \mathrm{pg} / \mathrm{mL}$. Los índices de manejo renal de fosfato y excreción urinaria de calcio fueron calculados según un método descrito (1) a partir de los niveles de calcio, fósforo y creatinina en orina de 24 horas. El calcio se midió con un método colorimétrico automatizado basado en la formación de un complejo calcio-o-cresoftaleina (2). La creatinina se determinó mediante una modificación del método de Jaffé (3) previamente descrito y comercializado. El TmPO4 fue estimado en el normograma de Bijvoet (4) a partir de niveles conocidos de fosfato sérico y reabsorción tubular de fosfato (valor normal para adultos 2,5-3,7). Los coeficientes de variación respectivos para la excreción urinaria de calcio Inter-intra-ensayo eran de 6,8 y 5,3 respectivamente y valor de referencia $0,1-0,2 \mathrm{mg} / \mathrm{GRF}$.

Se recogieron también los resultados de las técnicas de imagen procedentes de nuestros pacientes entre las que se hallaban la ecografía paratiroidea, la tomografía computerizada (TC) cérvico-torácica con contraste intravenoso, con secciones tomográficas cada $5 \mathrm{~mm}$ y la gamma grafía paratiroidea preoperatoria con 99Tc-Sestamibi, a dosis de $25 \mathrm{mCi}$, mediante gamma-cámara planar. Los registros de la gammagrafía se realizaron a los 10 minutos y a las $2 \mathrm{~h}$ post-inyección, utilizando colimador pin-hole y de agujeros paralelos. Se consideró patológica la persistencia a las $2 \mathrm{~h}$ del trazador en las imágenes registradas.

La densidad ósea mineral se midió mediante densitometría dual de rayos X ( DEXA) a nivel lumbar (L2-L4) y en cuello de fémur. Los datos fueron almacenados en la base de Microsoft ACCESS 97 y el análisis de los datos se realizó mediante estadística descriptiva (medias, frecuencias, desviación típica), el test de Student y el exacto de Fisher.

\section{RESULTADOS}

Fueron intervenidos 47 pacientes de HPTP, 89,4\% mujeres, con edad media $61,4 \pm 12,8$ años. La gran mayoría de los pacientes se encontraban asintomáticos $(45,7 \%)$ al diagnóstico, siendo este casual. Las manifestaciones clínicas (Fig. 1) fueron artralgias en el 75\%, en ningún caso se identificó osteoporosis o lesiones típicas de osteítis fibrosa, hipertensión arterial en el $37,5 \%$, anorexia y náuseas en el $8,3 \%$, ulcus en el $24,1 \%$, estreñimiento en el $33,3 \%$. En cuanto a síntomas urológicos destacaba el antecedente de nefrolitiasis en el $61,1 \%$ con predominio de oxalato cálcico. No se produjeron síntomas de hipercalcemia aguda.

Desde el punto de vista bioquímico, los valores medios de calcio preoperatorio fueron de 11,29 $\pm 0,78 \mathrm{mg} / \mathrm{dL}$, Fósforo

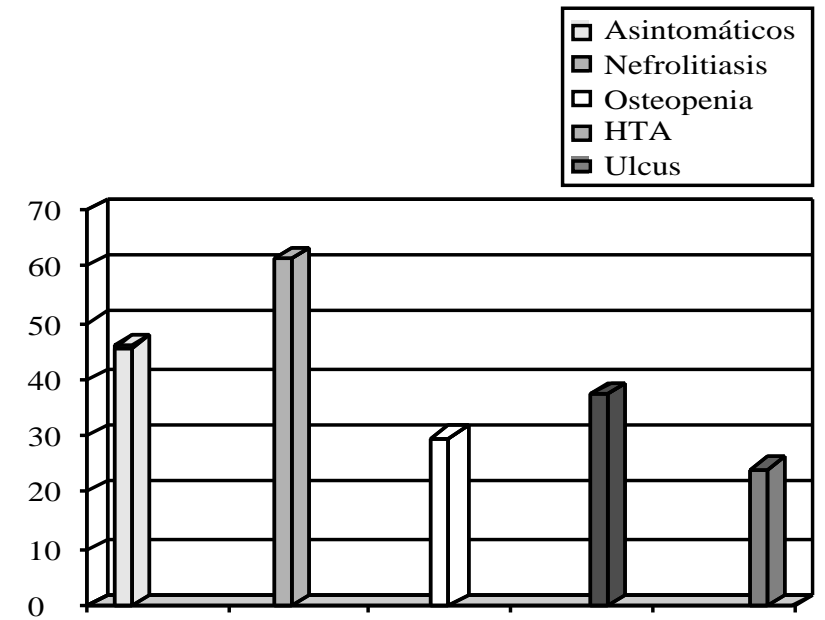

Fig. 1. Frecuencia de presentación de los síntomas en el HPTP.

2,46 $\pm 0,25 \mathrm{mg} / \mathrm{dL}$, PTH intacta de $136,3 \pm 72,3 \mathrm{pg} / \mathrm{ml}$, el transporte tubular medio de fosfatos fue $1,51 \pm 0,42 \mathrm{mg} / \mathrm{dL}$. Después de la intervención los valores medios de calcio fueron de 9,44 $\pm 0,68 \mathrm{mg} / \mathrm{dL}$, fósforo 2,98 $\pm 0,46 \mathrm{mg} / \mathrm{dL}$, PTH intacta $57,4 \pm 39,2 \mathrm{pg} / \mathrm{ml}$, transporte tubular medio de fosfatos $2,58 \pm 0,41 \mathrm{mg} / \mathrm{dL}$, con diferencias estadísticas significativas en todas ellas. En la Tabla I, se recogen todos los valores bioquímicos antes y después de la intervención y su significación estadística. La reducción del Z-score en el cuello del fémur fue del $87,5 \%$ (Z-score medio -0,53) y a nivel de L2-L4 del $83,6 \%$ (Z-score medio - 0,97 ), observándose una ligera reducción ósea sobretodo para hueso cortical (Fig. 2). Ninguno de los pacientes presentó osteoporosis definida mediante densitometría ósea, ni a nivel lumbar ni de cuello de fémur (todos tuvieron valores dentro de dos desviaciones típicas para su edad y sexo).

En cuanto a las técnicas de localización preoperatoria, 35 pacientes se hicieron la ecografía que mostró una sensibilidad del $66 \%$ y especificidad del $50 \%$, el TAC se llevó a cabo en 16 pacientes (sensibilidad del $46,1 \%$, especificidad $100 \%$ ) y la gammagrafía con sestamibi en 36 pacientes (sensibilidad $75 \%$ y especificidad $100 \%$ ). Hemos de considerar para el cálculo de estos datos, que incluimos sólo aquellos en los que quirúrgicamente se encontró patología paratiroidea. En la figura 3 se muestra la gammagrafía de un adenoma paratiroideo en la fase de captación precoz del trazador y en la figura 4 en la fase tardía a los 120 minutos.

El diagnóstico anatomopatológico fue el de adenoma en $40 / 47$ casos $(85,1 \%)$, de hiperplasia en $6(12,7 \%)$ y carcinoma en uno $(2,1 \%)$. En ninguno de los pacientes se produjeron complicaciones importantes derivadas de la cirugía, aunque cabe destacar el hipoparatiroidismo en uno de los enfermos. Se consiguió la curación en todos, excepto en uno en el cual no se localizó la glándula patológica.

\section{DISCUSIÓN}

Actualmente el HPTP es una enfermedad relativamente común que se identifica en la mayoría de los casos por la determinación de calcio plasmático en análisis de rutina (5-7). Así sucede en los pacientes que hemos estudiado en los últimos cinco años y aunque el $54,3 \%$ de los mismos presentaba 
TABLA I

VALO RES BIO Q UÍM ICO S DE LOS PACIENTES ANTES Y DESPUÉS DE LA INTERVENCIÓ N Q UIRÚRGICA

\begin{tabular}{lccccc}
\hline & Rango & Preoperatorio & Postoperatorio & \\
& Unidades & normal & $x \pm S D$ & $x \pm S D$ & $p$ \\
\hline Calcemia & $\mathrm{mg} / \mathrm{dL} \pm \mathrm{DS}$ & $8,4-10,2$ & $11,29 \pm 0,78$ & $9,44 \pm 0,68$ & $<0,001$ \\
Fosforemia & $\mathrm{mg} / \mathrm{dL} \pm \mathrm{DS}$ & $2,5-4,5$ & $2,46 \pm 0,25$ & $2,98 \pm 0,46$ & $<0,05$ \\
PTH intacta & $\mathrm{pg} / \mathrm{ml} \pm \mathrm{DS}$ & $20-70$ & $136,29 \pm 72,3$ & $57,4 \pm 39,2$ & 0,002 \\
TmPO 4 & $\mathrm{mg} / \mathrm{dL}$ & $2,5-3,7$ & $1,51 \pm 0,42$ & $2,58 \pm 0,41$ & $<0,001$ \\
Calciuria & $\mathrm{mg} / \mathrm{GRF}$ & $0,1-0,2$ & $0,26 \pm 0,11$ & $\mathrm{NR}$ & \\
RTP & $\%$ & $80-86$ & $68,3 \pm 15,7$ & $\mathrm{NR}$ & \\
Fosfatasa alcalina & $\mathrm{UI} / \mathrm{L}$ & $80-300$ & $270,3 \pm 93$ & $\mathrm{NR}$ & \\
\hline
\end{tabular}

TmPO 4: tasa máxima de transporte tubular de fosfatos. RTP: reabsorción tubular de fosfatos. La calciuria se ha corregido para creatinina en orina de 24 horas; NR: no disponemos de resultados; Tm/P: transporte medio tubular de fosfatos; excreción urinaria de calcio corregido para creatinina orina de 24 horas; RTP: reabsorción tubular de fosfatos; FA: fosfatasa alcalina.

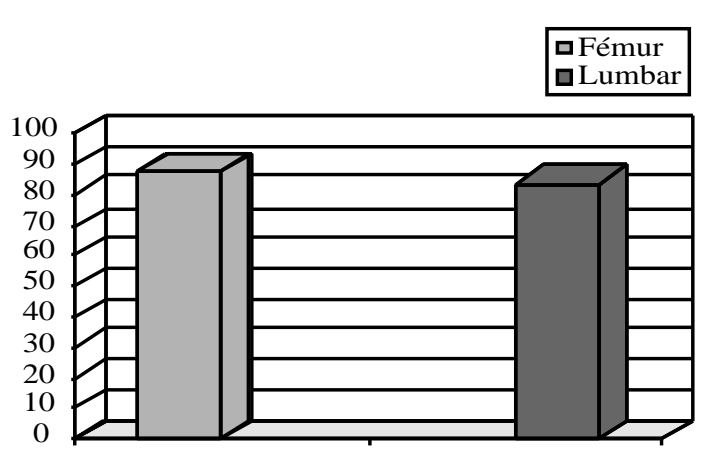

Fig. 2. Densitometría ósea.

algún síntoma sutil, ninguno de ellos tenían las manifestaciones clínicas clásicas del HPTP como la enfermedad renal (cálculos renales, disminución de la función renal y nefrocalcinosis) o la ósea (cráneo en sal y pimienta, reabsorción de las falanges distales, quistes óseos).

Actualmente, a parte del diagnóstico a través de la medición de la calcemia, se puede sospechar por la osteoporosis $2^{\circ}$. En nuestra serie, ninguno de los pacientes presentó osteoporosis definida por densitometría, pero sí observamos reducción de la masa ósea para su misma edad y sexo, sobretodo a nivel de hueso cortical (sobretodo a nivel de fémur), aunque quizás menos de lo que cabría esperar (8). Es conocido que la osteopenia mejora tras la intervención quirúrgica (9), dato que no hemos podido comprobar en nuestro estudio por carecer en la mayoría de los casos de densitometría postintervención. En otras ocasiones, el diagnóstico se sospecha por nefrolitiasis, fundamentalmente de oxalato cálcico. La frecuencia de nefrolitiasis también ha disminuido, así en un estudio (10) de 197 pacientes en 1981 , el $51 \%$ presentaban nefrolitiasis mientras que actualmente está alrededor del 20\% (11). En nuestro serie los pacientes tenían nefrolitiasis en el $61,1 \%$. Más raramente se les diagnostica por síntomas digestivos, como ulcus y pancreatitis, o por hipertensión arterial, de hecho algunos trabajos ponen en duda que los pacientes con HPTP presenten HTA (12). En nuestra caso se detectó HTA en el 37,5\% en pacientes con una edad media de 61 años, siendo similar la prevalencia de hipertensión para ese grupo de edad. Sin embargo, en uno de nuestros pacientes en tratamiento antihipertensivo con tres fármacos se curó la hipertensión tras la intervención quirúrgica, no mejorando en ninguno de los otros.
Entre las determinaciones bioquímicas, en la actualidad se define el hiperparatirodismo como el aumento de la PTHi con hipercalcemia, siendo raros los casos en que se precisan determinaciones seriadas de calcio por hipercalcemia intermitente, por otra parte, es conveniente descartar entidades como la hipercalciuria familiar benigna o la hipercalcemia inducida por litio. En todos nuestros pacientes hallamos valores de calcio por encima de la normalidad, así como de TPI y valores bajos de fósforo debido al efecto fosfatúrico de la PTH. La intervención quirúrgica permitió la corrección de la hipercalcemia en todos los casos. En cuanto a la localización preoperatoria del HPTP existe discusión sobre la rentabilidad de las pruebas diagnósticas, de hecho, se acepta que en el 95\% de las exploraciones quirúrgicas iniciales de cuello, realizadas por un cirujano experto, identifica y elimina la lesión paratiroidea (13). Esta afirmación, unida a la baja sensibilidad (60-75\%) (en nuestra serie de pacientes obtuvimos una sensibilidad similar, siendo algo superior para la ecografía (66\%) que para la TC $(46,1 \%)$ y a los falsos positivos $(15 \%)$ con la ecografía, tomografía computerizada, resonancia magnética, gammagrafía de sustracción, arteriografía y cateterismo venoso, hacen dudar del valor diagnóstico de dichas técnicas $(13,14)$.

Sin embargo, a partir de 1989 (15) con la introducción de la gammagrafía de paratiroides con 99Tc-sestamibi, se diagnostica el HPTP con una sensibilidad y especificidad cercanas al 90\% (16-19), aunque en algunas otras series es algo inferior $(20,21)$, como sucedió en nuestro caso. La sensibilidad de la técnica parece ser inferior para las glándulas hiperplásicas que para los adenomas $(19,20)$. Una limitación de la técnica son los falsos positivos que se pueden producir y que parecen más frecuentemente debidos a la coexistencia de bocio nodular (22). A pesar de estas consideraciones, la gammagrafía es la técnica que permite la detección de glándulas ectópicas (causa frecuente del fracaso de intervenciones quirúrgicas sin localización preoperatoria) y ,por otro lado, el control de la persistencia o de las recidivas tras la cirugía.

El tratamiento del HPTP es controvertido, ya que no existe un tratamiento médico eficaz y en la actualidad no conocemos bien la historia natural de la enfermedad en pacientes asintomáticos. Por ello, en 1990 la Conferencia del manejo del paciente con HPTP (23) recomendó intervención quirúrgica en las siguientes circunstancias: 1) Si la concentración de Ca sérico está notablemente aumentada (11,4-12 $\mathrm{mg} / \mathrm{dL}$ ). 2) si se ha presentado algún episodio previo de 

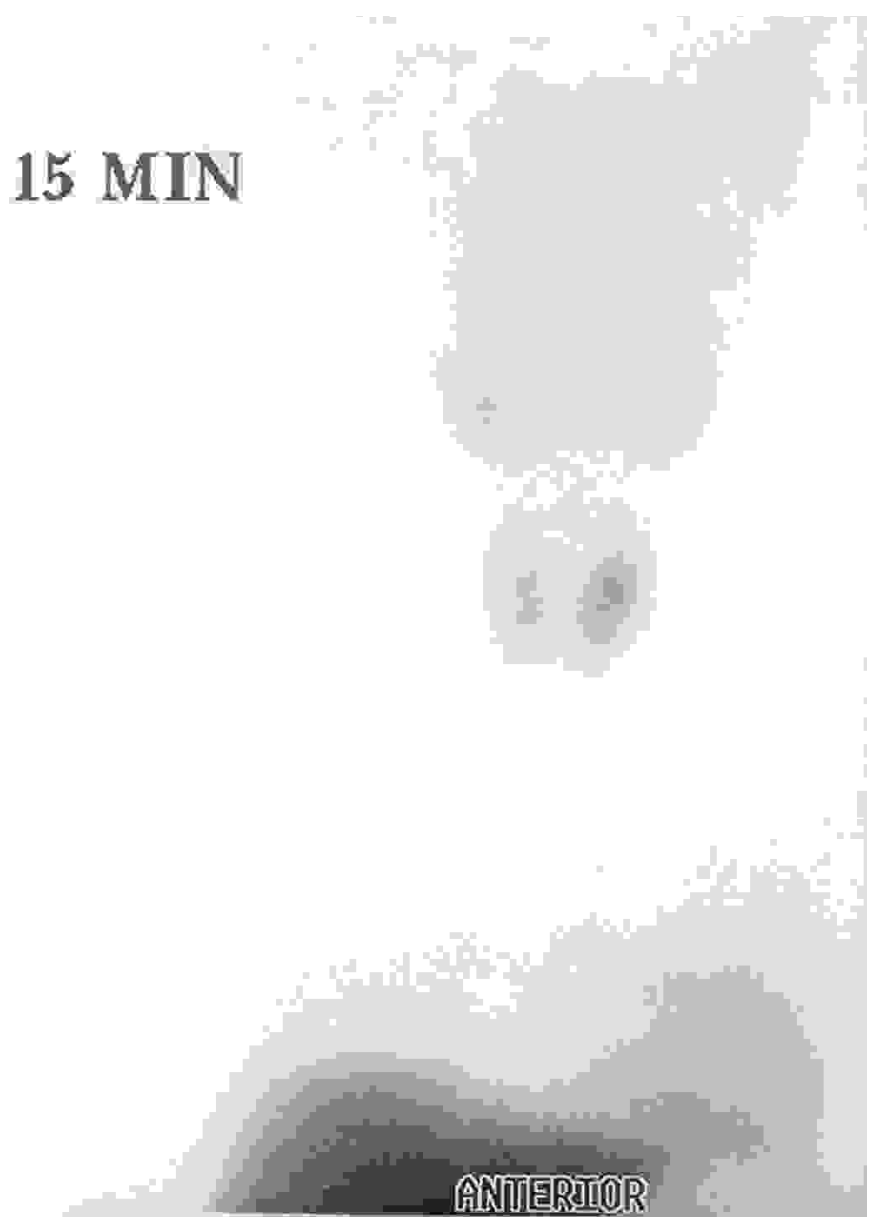

Fig. 3. Gammagrafía de adenoma paratiroideo. Fase precoz.

hipercalcemia que ponga en peligro la vida. 3) Si el aclaramiento de creatinina se reduce en un $30 \%$ con respecto a las personas de la misma edad. 4) Si existe un cálculo renal. 5) Si el calcio urinario está notablemente aumentado (>400 $\mathrm{mg} / 24 \mathrm{~h})$. 6) Si la masa ósea se encuentra muy disminuida (menos de 2 desviaciones estándar por debajo de lo normal para la edad el sexo y la raza). 7) Si es un paciente joven (menor de 50 años o es una mujer premenopáusica). Un estudio reciente de Silverberg et al (9) nos clarifica mejor la evolución de los pacientes con HPTP asintomáticos. En este estudio de 10 años de seguimiento, los pacientes que se intervinieron consiguieron normalizar los niveles de calcio sérico e incrementaron la densidad ósea ( $8 \%$ en el primer año y de $12 \%$ a los 10 años en la zona lumbar; y $6 \%$ en el

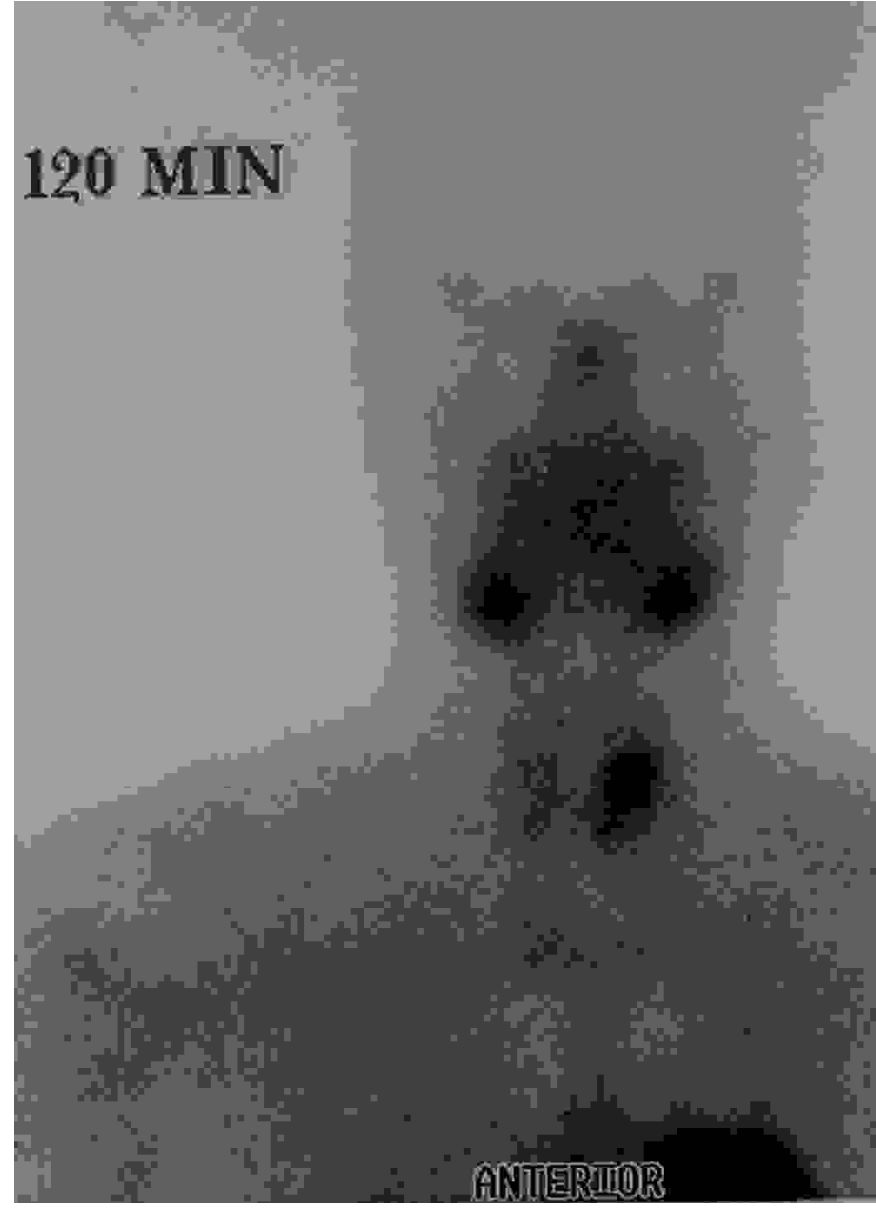

Fig. 4. Gammagrafía de adenoma paratiroideo. Fase tardía.

primer año y $14 \%$ a los 10 años a nivel de fémur). Una cuarta parte de los pacientes no intervenidos presentó progresión de la enfermedad en algún momento de su evolución (con criterio definido de intervención quirúrgica). En nuestra serie los pacientes se intervinieron por presentar alguna de las indicaciones recomendadas. Ninguno tuvo lesión del nervio laríngeo inferior y tan solo uno desarrolló hipoparatiroidismo permanente.

En conclusión, el HPTP presenta en la actualidad escasos síntomas. Principalmente se trata de adenomas. La técnica de localización preoperatoria más útil es la gammagrafía con 99mTc-sestamibi (sobre todo indicada en glándulas ectópicas y en permanencia del HPTP o recidiva) y el tratamiento definitivo es la intervención quirúrgica.

\section{Bibliografía}

1. Broadus AE. Metabolismo mineral. En: Felig P et al, eds. Endocrinología y metabolismo. Madrid: McGraw-Hill; 1981: 1024-139.

2. Gosling P. Analytical reviews in clinical biochemistry: Calcium measurement. Ann Clin Biochem 1986; 23: 146-1 51.

3. Bartels H, Bölmer H, Heierll C. Serum creatinine determination without protein precipitation. Clin Chim Acta 1972; 37: 193-7.

4. De Walton RJ, Bijvoet QL. Nomogram for determination of renal threshold for phosphate concentration. Lancet 1975; 2: 309.

5. Barbier J, Kraimps JL, Denizot A, Henry JF. Aspect chiruricale actuel de l'hyperparathyroidisme primaire (cent ans apres F. D. Von Reklinghausen). Chirurgie 1992; 118; 439-47.

6. Lundgren E, Gillot AR, Wiseman JS, Beck J. The role of preoperative localization in primary hyperparathyroidism. The American Surgeon 1995; 61: 394-6.

7. Mundy GR, Cove DH, Fisken R. Primary hyperparathyroidism: changes in the pattern of clinical presentation. Lancet 1980; 1: 1317-20. 
8. Muñoz-Torres M, Ventosa Viñas M, Mezquita Raya P et al. Utilidad de la densitometría ósea en la evaluación del hiperparatiroidismo primario. Med Clin (Barc) 2000; 114: 521-4.

9. Silverberg SJ, Shane E, Jacobs TP, Siris E, Bilezikian JP. A 10-year prospective study of primary hyperpartathyroidism with or without parathyroid surgery. N Engl J Med 1999; 341: 1249-55.

10. Scholz DA, Purnell DC. Asyntomatic primary hyperparathuroidism: 10year prospective study. Mayo Clin Proc 1981; 56: 473-8.

11. Bilezikian JP, Silverberg SJ, Gartenberg F et al. Clinical presentation of primary hyperparathyroidism. In: Bilezikian JP, Ed. The parathyroidis: basic and clinical concepts. New York: Raven Press, 1994: 457-70.

12. Strewler GJ. Metabolismo mineral y enfermedad ósea metabólica. En Greenspan FS and Strewler GJ. Endocrinología básica y clínica. Mexico ed. El Manual Moderno,1998: 299-360.

13. Norton JA, Brennan MF, Wells SA Jr. Surgical management of hyperparathyroidism. En Bilezikian JP, Marcus R, Levine MA, editores. The parathyroids, basic and clinical concepts. Nueva York: Raven Press 1994; 531-51.

14. Doppman JL. Preoperative localization of parathyroid tissue in primary hyperparthyroidism. En: Bilezikian JP, Marcus R, Levine MA, editores. The parathyroids, basic and clinical concepts. Nueva York: Raven Press 1994; 553-65.

15. Coalkley AJ, Kettle AG, Wells CP, O`Doherty MJ, Collins REC. 99 Tsestamibi new agent for parathyroid imaging. Nucl Med Commun 1989; 10:791-4.

16. Weber CJ, Vansant J, Alazraki N, Christy J, Watts N, Philips LS et al. Value of technetium 99m-sestamibi iodine 123 imbaging in preoperative parathyroid surgery. Surgery $1993 ; 114: 1011-8$.
17. Thulé P, Thakakore K, Vansant J, McGarity W, Weber C, Philips LS Preoperative localization of parathyroid tissue with technetium-99-msestamibi 123I subtraction scanning. J Clin Endocrinol Metab 1994; 78 77-82.

18. Johnson LB, Carroll MJ, Britton KE, Lowe DG, Shand W, Besser GM et al. The accuracy of parathyroid gland localization in primary hyperparthyroidism using sestamibi radionuclide imaging. J Clin Endocrinol Metab 1996; 81: 346-52.

19. Berná LL, Piera J, Rodríguez-Espinosa J, Caixás A, Puig-Domingo M, Farrerons $\mathrm{J}$ et al. Exploración isotópica con 99mTc-sestamibi en doble fase en la localización de lesiones de las glándulas paratiroides. Med Clin 1999; 112: 201-5.

20. Piñero Madrona A, Rodríguez González JM, Ortíz Sebastian S, Parrilla Paricio P. Diagnóstico de localoización de las glándulas patológicas en el hiperparatiroidismo primario: comparación de la gammagrafía con 99-mTc-sestamiibi, la ecografía y la tomografía computerizada. Med Clin (Barc) 1998; 111: 558-559.

21. Villar G, Ugarte Abásolo E, Echevarría A, Vázquez JA. Utilidad de los estudios de localización preoperatorios en la intervención quirúrgica inicial del hiperparatiroidismo primario. Med Clin 1998; 110 : $285-9$

22. Geatti O, Shapiro B, Orsolon PG, Proto G, Guerra UP, Antonucci F et al. Localization of parathyroid enlargement: experience with technetium$99 \mathrm{~m}$ methoxyisobutyl isonitrile and thalium 201 scintigraphy, ultrasonography and computed tomography. Eur J Nucl Med 1994; 219: 582-6.

23. NIH conference: diagnosis and management of asymptomatic primary hyperparathyroidism: consensus development conference statement. Ann Intern Med 1991; 114: 593-7. 